\title{
BECKMANN-TYPE PROBLEM FOR DEGENERATE HAMILTON-JACOBI EQUATIONS
}

\author{
HAMZA ENNAJI ${ }^{\dagger}$, NOUREDDINE IGBIDA ${ }^{\dagger}$, AND VAN THANH NGUYEN $^{\ddagger}$
}

\begin{abstract}
The aim of this note is to give a Beckmann-type problem as well as corresponding optimal mass transportation associated with a degenerate Hamilton-Jacobi equation $H(x, \nabla u)=$ 0 , coupled with non-zero Dirichlet condition $u=g$ on $\partial \Omega$. In this article, Hamiltionian $H$ is continuous in both arguments, coercive and convex in the second, but not enjoying any property of existence of a smooth strict sub-solution. We also provide numerical examples.
\end{abstract}

\section{INTRODUCTION}

Hamilton-Jacobi (HJ for short) equation, coupled with a Dirichlet condition, is a first order equation of the type

$$
\begin{cases}H(x, \nabla u(x))=0 & \text { in } \Omega \\ u=g & \text { on } D \subset \bar{\Omega} .\end{cases}
$$

It has attracted the attention of mathematicians and physicists since it appears in many fields, in particular, classical mechanics, geometry, seismic analysis, optics, image processing, etc. Our main interest here lies in the study of the connection between these types of equation and the Beckmann problem as well as the Monge-Kantorovich problem in the case where $H$ is continuous in both arguments, coercive and convex in the second, but not enjoying any property of existence of a smooth strict sub-solution. Recall that Beckmann's problem (called by Beckmann a continuous model of transportation [1]) is a divergence PDE-constrained optimization problem. It is important in the study of transportation activities. As to the Monge-Kantorovich problem, it consists in finding the best way to push forward between two given measures (usually called goods and consumers) related to some cost function. It is a linear optimization problem which appears in the study of optimal transportation and allocation of resources.

1.1. Beckmann's problem and optimal transport. Beckmann's problem appears typically in the study of optimal transference between two distributions of masses. For instance in urban area represented by a bounded domain $\Omega \subset \mathbb{R}^{N}, N=2$, it can describe some kind of optimal traffic between two distributions of residents and services. These distributions may be represented by two given nonnegative Radon measures $\mu_{1}$ and $\mu_{2}$, respectively. So, the signed measure

Date: October 10, 2020.

${ }^{\dagger}$ Institut de recherche XLIM, UMR-CNRS 7252, Faculté des Sciences et Techniques, Université de Limoges, France.

Emails: hamza.ennaji@unilim.fr, noureddine.igbida@unilim.fr.

${ }^{\ddagger}$ Department of Mathematics and Statistics, Quy Nhon University, Vietnam.

Email: nguyenvanthanh@qnu.edu.vn. 
$\mu:=\mu_{2}-\mu_{1}$ represents the local measure of excess demand. The consumers traffic is given by a traffic flow field, i.e. a vector field $\Phi: \Omega \rightarrow \mathbb{R}^{N}$ whose direction indicates the consumers' travel direction and whose modulus $|\Phi|$ is the intensity of the traffic. The relationship between the excess demand and the traffic flow is obtained from the equilibrium condition:

$$
-\operatorname{div}(\Phi)=\mu \quad \text { in } \mathcal{D}^{\prime}(\Omega)
$$

This condition describes some kind of equilibrium: the outflow of consumers equals the excess demand in any subregion $\omega \subset \Omega$, i.e. $\int_{\partial \omega} \Phi \cdot n \mathrm{~d} s=\mu(\omega)$. In standard Beckmann's problem, the measures $\mu_{1}$ and $\mu_{2}$ have equal masses (a balanced condition between residents and services), and the urban area is assumed to be isolated, i.e. no traffic flow should cross the boundary of the city $\Omega$. In other words, $\Phi$ is subject to the boundary condition

$$
\Phi \cdot n=0 \quad \text { on } \partial \Omega
$$

where $n$ denotes the outward normal vector to the boundary. Assuming the transportation cost per consumer is given by the quantity $F(x, \Phi(x))$, for a given function $F: \Omega \times \mathbb{R}^{N} \rightarrow \mathbb{R}^{+}$, Beckmann therefore argued that one may define the transportation cost between $\mu_{1}$ and $\mu_{2}$ as the infimum of the total cost of the traffic $\int_{\Omega} F(x, \Phi(x)) \mathrm{d} x$.

Recall that this problem is tightly related to the Monge-Kantorovich problem (also called optimal transport) which consists in finding the best way to push forward the measure $\mu_{1}$ into $\mu_{2}$ related to a specified cost function. For $i=1,2$, we use the convention of denoting by $\pi_{i}$ : $\mathbb{R}^{N} \times \mathbb{R}^{N} \rightarrow \mathbb{R}^{N}, i=1,2$ the projections: $\pi_{1}(x, y):=x, \pi_{2}(x, y):=y$. Given a nonnegative Radon measure $\mu$ on $\mathbb{R}^{N} \times \mathbb{R}^{N}$, its marginals are measures defined by $\operatorname{proj}_{x}(\mu):=\pi_{1} \# \mu, \operatorname{proj}_{y}(\mu):=$ $\pi_{2} \# \mu$, i.e. $\operatorname{proj}_{x}(\mu)(A):=\mu\left(\pi_{1}^{-1}(A)\right)=\mu\left(A \times \mathbb{R}^{N}\right)$ and $\operatorname{proj}_{y}(\mu)(B)=\mu\left(\mathbb{R}^{N} \times B\right)$ for any Borel subsets $A, B \subset \mathbb{R}^{N}$. Then, the Monge-Kantorovich (MK) problem consists in finding a nonnegative Radon measure $\mu$ on $\mathbb{R}^{N} \times \mathbb{R}^{N}$ such that $\pi_{1} \# \mu=\mu_{1}, \pi_{2} \# \mu=\mu_{2}$ and minimizing the total work

$$
\iint c(x, y) \mathrm{d} \mu(x, y)
$$

where $c$ is a given ground cost function describing the charge one needs to pay for transporting one unit of $\mu_{1}$ into a unit of $\mu_{2}$. A minimizer $\mu$ of the above problem is called an optimal plan. One of basic concepts in the optimal transport theory is the Kantorovich duality that can be restated as follows (see e.g. [23, Chapter 5]):

Theorem 1.1. Let $c$ be a lower semicontinuous (l.s.c.) cost function and $\mu_{1}, \mu_{2} \in \mathcal{M}_{b}^{+}\left(\mathbb{R}^{N}\right)$ be nonnegative Radon measures such that $\mu_{1}\left(\mathbb{R}^{N}\right)=\mu_{2}\left(\mathbb{R}^{N}\right)$. Then

(i) The (MK) problem has an optimal plan and the Kantorovich duality holds true, i.e.

$\min \left\{\iint c(x, y) \mathrm{d} \mu: \pi_{1} \# \mu=\mu_{1}, \pi_{2} \# \mu=\mu_{2}\right\}=\sup \left\{\int u \mathrm{~d} \mu_{1}+\int v \mathrm{~d} \mu_{2}:(u, v) \in \mathcal{S}_{c}\left(\mu_{1}, \mu_{2}\right)\right\}$,

where

$$
\mathcal{S}_{c}\left(\mu_{1}, \mu_{2}\right):=\left\{(u, v) \in L_{\mu_{1}}^{1}\left(\mathbb{R}^{N}\right) \times L_{\mu_{2}}^{1}\left(\mathbb{R}^{N}\right): u(x)+v(y) \leq c(x, y) \forall x, y \in \mathbb{R}^{N}\right\} .
$$


(ii) If $c(x, y) \leq C_{\mu_{1}}(x)+C_{\mu_{2}}(y)$ for some $\left(C_{\mu_{1}}, C_{\mu_{2}}\right) \in L_{\mu_{1}}^{1} \times L_{\mu_{2}}^{1}$, then the dual problem on the right-hand side (called Kantorovich dual problem) has an optimal solution.

(iii) If the cost function $c$ satisfies the triangle inequality and $c(x, x)=0$ for any $x \in \mathbb{R}^{N}$, then the Kantorovich dual problem can be rewritten by using one variable u only:

$$
\sup \left\{\int_{\mathbb{R}^{N}} u \mathrm{~d}\left(\mu_{2}-\mu_{1}\right): u \in L_{\mu_{1}}^{1} \cap L_{\mu_{2}}^{1} \text { and } u(y)-u(x) \leq c(x, y) \forall x, y \in \mathbb{R}^{N}\right\} .
$$

The connection between Beckmann's problem and the Monge-Kantorovich problem is well known, for instance, in the case where $c$ is given by the distance $d_{k}$ associated with the Riemannian metric $k$, i.e.

$$
d_{k}(x, y)=\inf \left\{\int_{0}^{1} k(\varphi(t))\left|\varphi^{\prime}(t)\right| \mathrm{d} t: \varphi \in \operatorname{Lip}([0,1], \bar{\Omega}), \varphi(0)=x, \varphi(1)=1\right\} .
$$

In this case, since $c=d_{k}$ is a distance, the Kantorovich dual problem reads as (see Theorem 1.1)

$$
\max \left\{\int_{\bar{\Omega}} u \mathrm{~d}\left(\mu_{2}-\mu_{1}\right): u(y)-u(x) \leq d_{k}(x, y) \text { for all } x, y \in \bar{\Omega}\right\},
$$

or equivalently,

$$
\max \left\{\int_{\bar{\Omega}} u \mathrm{~d}\left(\mu_{2}-\mu_{1}\right):|\nabla u(x)| \leq k(x) \text { for all } x \in \Omega\right\} .
$$

Then, using Fenchel-Rockafellar duality, one leads to another dual problem to (1.4):

$$
\inf \left\{\int_{\Omega} k(x)|\Phi|(x) \mathrm{d} x: \Phi \in L^{1}(\Omega)^{N},-\operatorname{div}(\Phi)=\mu_{2}-\mu_{1} \text { in } \mathcal{D}^{\prime}\left(\mathbb{R}^{N}\right)\right\} .
$$

This is Beckmann's problem with $F(x, p)=k(x)|p|$. For the general case of non-degenerate Finsler metric $F$, one has (see e.g. [3, 17, 21, 18]), with $d_{F}(x, y):=\inf _{\substack{\varphi \in \operatorname{Lip}([0,1], \bar{\Omega}) \\ \varphi(0)=x, \varphi(1)=1}} \int_{0}^{1} F\left(\varphi(t), \varphi^{\prime}(t)\right) \mathrm{d} t$,

$$
\begin{aligned}
& \min \left\{\iint_{\bar{\Omega} \times \bar{\Omega}} d_{F}(x, y) \mathrm{d} \mu: \pi_{1} \# \mu=\mu_{1}, \pi_{2} \# \mu=\mu_{2}\right\} \\
& =\max \left\{\int_{\bar{\Omega}} u \mathrm{~d}\left(\mu_{2}-\mu_{1}\right): u(y)-u(x) \leq d_{F}(x, y) \text { for all } x \in \bar{\Omega}\right\} \\
& =\inf \left\{\int_{\Omega} F(x, \Phi(x)) \mathrm{d} x: \Phi \in L^{1}(\Omega)^{N},-\operatorname{div}(\Phi)=\mu_{2}-\mu_{1} \text { in } \mathcal{D}^{\prime}\left(\mathbb{R}^{N}\right)\right\} .
\end{aligned}
$$

1.2. Hamilton-Jacobi equation and Beckmann's problem. The connection between HJ equation coupled with a Dirichlet condition (1.1) and Beckmann's problem is not straightforward.

In the case where $H(x, p)=|p|-k(x)$ (i.e., the Eikonal equation $|\nabla u(x)|=k(x)$ ) complemented with Dirichlet boundary condition $u=g$ on $\partial \Omega$, the viscosity solution of (1.1) can be characterized through the following optimization problem

$$
\max \left\{\int_{\Omega} u \mathrm{~d} x: u(x)-u(y) \leq d_{k}(y, x) \text { and } u=g \text { on } \partial \Omega\right\},
$$


where $d_{k}$ is given by (1.2). Thanks to (1.3), it is meaningful to consider this problem as some kind of push-forward of $\mu_{1}:=\mathcal{L}_{\mid \Omega}^{N}$ the restriction of Lebesgue measure on $\Omega$. The offset is clearly connected to the lack of a measure $\mu_{2}$ which can fit out the problem with the balanced property. Actually, the linked Beckmann and Monge-Kantorovich problems aim to find moreover the optimal $\mu_{2}$ concentrated on $\partial \Omega$ which will consume $\mu_{1}:=\chi_{\Omega}$. More precisely, we will prove that the problem is connected to

$$
\min _{\nu \in \mathcal{M}_{b}(\partial \Omega)} \max _{u}\left\{\int_{\Omega} u(\mathrm{~d} x-\mathrm{d} \nu)-\int_{\partial \Omega} g \mathrm{~d} \nu: u \text { is } 1-\text { Lipschitz w.r.t } d_{k}\right\}
$$

which provides the following modified Beckmann problem

$$
\min _{\nu \in \mathcal{M}_{b}(\partial \Omega)} \inf _{\Phi \in L^{1}(\Omega)^{N}}\left\{\int_{\Omega} k(x)|\Phi|(x) \mathrm{d} x+\int_{\partial \Omega} g \mathrm{~d} \nu:-\operatorname{div}(\Phi)=1-\nu \operatorname{in} \mathcal{D}^{\prime}\left(\mathbb{R}^{N}\right)\right\} .
$$

as well as to the following modified Monge-Kantorovich problem

$$
\min _{\gamma \in \mathcal{M}^{+}(\bar{\Omega} \times \bar{\Omega}), \nu \in \mathcal{M}_{b}(\partial \Omega)}\left\{\int_{\bar{\Omega} \times \bar{\Omega}} d_{k}(x, y) \mathrm{d} \gamma(x, y)+\int_{\partial \Omega} g \mathrm{~d} \nu: \pi_{1} \sharp \gamma=\nu^{+}, \pi_{2} \sharp \gamma=1+\nu^{-}\right\} .
$$

For the general case of degenerate Hamiltonian $H$, we will also obtain the corresponding results by means of the intrinsic distance $d_{\sigma}$ associated to the Hamiltonian $H$ (see (2.7)).

The main contribution of our paper is the rigorous treatment for the case of degenerate Hamiltionian $H$ and its degenerate intrinsic metric $d_{\sigma}$, as well as non-zero Dirichlet condition. Moreover, we also illustrate numerical examples.

This paper is organized as follows. In Section 2 we recall briefly some notions around HJ equation as well as the main results establishing the connection between HJ equation and Beckmanntype problem. Section 3 is dedicated to the proofs of the main results. Numerical examples are presented in Section 4 . Finally, Section 5 contains some comments and extensions.

\section{Preliminaries AND MAin RESUlts}

2.1. Preliminaries. All the properties and notions introduced in this subsection are more or less known. Proofs and more details can be found in [6, 19, 22,

Given a regular connected open domain $\Omega \subset \mathbb{R}^{N}$, and a continuous Hamiltonian $H: \bar{\Omega} \times \mathbb{R}^{N} \rightarrow$ $\mathbb{R}$ satisfying, for any $x \in \bar{\Omega}, Z(x):=\left\{p \in \mathbb{R}^{N}, H(x, p) \leq 0\right\}$ :

(H1) coercivity: $Z(x)$ is compact;

(H2) convexity: $Z(x)$ is convex;

(H3) $H(x, 0) \leq 0$, i.e. $0 \in Z(x)$,

we consider the following HJ equation

$$
H(x, \nabla u)=0, x \in \Omega .
$$

We recall briefly some definitions related to viscosity theory. A continuous function $u: \Omega \rightarrow \mathbb{R}$ is said to be a viscosity subsolution (respectively supersolution) of 2.6 if $H(x, \nabla \phi(x)) \leq 0$ (respectively $H(x, \nabla \phi(x)) \geq 0$ ) for any $x \in \Omega$ and any $C^{1}$ function $\phi$ such that $u-\phi$ has a strict local maximizer (respectively minimizer) at $x$. Finally, $u$ is a viscosity solution of (2.6) if it is both a subsolution and a supersolution. We denote by $\mathcal{S}_{H}^{-}(\Omega)$ (respectively $\mathcal{S}_{H}^{+}(\Omega)$ ) the family of viscosity subsolutions (respectively supersolutions) of (2.6). 
For $x \in \bar{\Omega}$, we define the support function of the 0 -sublevel set $Z(x)$ by

$$
\sigma(x, q):=\sup q \cdot Z(x)=\sup \{q \cdot p \mid p \in Z(x)\} \text { for } q \in \mathbb{R}^{N} .
$$

The assumption (H1)-(H3) ensures that $\sigma$ is a possibly degenerate Finsler metric, i.e., $\sigma$ is a continuous nonnegative function in $\bar{\Omega} \times \mathbb{R}^{N}$, convex and positively homogeneous with the second variable $q$. Due to the assumption (H3), $\sigma(x, q)$ is possible to equal to 0 for $q \neq 0$, which leads to the degeneracy and its dual $\sigma^{*}$, as defined below, may take the value $+\infty$. Here, the dual $\sigma^{*}$ (also called polar) is defined by

$$
\sigma^{*}(x, p):=\sup _{q}\{p \cdot q \mid \sigma(x, q) \leq 1\} .
$$

Under the assumption (H1)-(H3), it is not difficult to see that there exists $K>0$

$$
0 \leq \sigma(x, q) \leq K|q| \text { for } x \in \bar{\Omega}, q \in \mathbb{R}^{N} \text {. }
$$

We denote by $\Gamma(x, y)$ the set of Lipschitz curves defined on $[0,1]$ joining $x, y$ in $\bar{\Omega}$. We then define the intrinsic distance by

$$
d_{\sigma}(x, y):=\inf _{\zeta \in \Gamma(x, y)} \int_{0}^{1} \sigma(\zeta(t), \dot{\zeta}(t)) \mathrm{d} t
$$

which is a quasi-distance, i.e. satisfying $d_{\sigma}(x, x)=0$ and the triangular inequality, but not necessarily symmetric. We summarize some basic characterizations of subsolution in terms of intrinsic distance $d_{\sigma}$.

Proposition 2.2. (12])

1) $d_{\sigma}$ is a quasi-distance in the sense that for any $x, y \in \bar{\Omega} d_{\sigma}(x, y) \geq 0$ and $d_{\sigma}(x, x)=0$. Moreover, for all $x, y, z \in \bar{\Omega}$ one has $d_{\sigma}(x, y) \leq d_{\sigma}(x, z)+d_{\sigma}(z, y)$.

2) For any $x \in \Omega$ one has $d_{\sigma}(x,.) \in \mathcal{S}_{H}^{-}(\Omega) \cup \mathcal{S}_{H}^{+}(\Omega \backslash\{x\})$.

3) Compatibility condition: $v \in \mathcal{S}_{H}^{-}(\Omega)$ if and only if $v(x)-v(y) \leq d_{\sigma}(y, x)$ for any $x, y \in \Omega$.

Given a closed subset $D \subset \bar{\Omega}$ (typically $D=\partial \Omega$ or $D=\bar{\omega}$ for some $\omega \subset \subset \Omega$ ), we consider the following HJ equation

$$
\begin{cases}H(x, \nabla u)=0 & \text { in } \Omega \\ u=g & \text { on } D\end{cases}
$$

where $g: D \rightarrow \mathbb{R}$ is a continuous function satisfying the compatibility condition

$$
g(x)-g(y) \leq d_{\sigma}(y, x) \quad \text { for any } x, y \in D .
$$

Thanks to Proposition 2.2, the unique maximal viscosity subsolution of the equation (2.8) can be recovered via the following maximization problem

$$
\max \left\{\int_{\Omega} u \mathrm{~d} x: u(x)-u(y) \leq d_{\sigma}(y, x), \forall x, y \in \Omega \text { and } u=g \text { on } D\right\},
$$

where $d_{\sigma}(.,$.$) is the intrinsic distance associated to the Hamiltonian defined by (2.7).$ 
Note that in the case of non-degenerate Finsler metric $\sigma$ (i.e., $H(x, 0)<0$ ), the 1 -Lipschitz condition $u(x)-u(y) \leq d_{\sigma}(y, x)$ for all $x, y \in \Omega$ can be characterized by $u \in W^{1, \infty}(\Omega)$, $\sigma^{*}(x, \nabla u(x)) \leq 1$ a.e. $x \in \Omega$. This property still holds true for degenerate Hamiltionians $H$ (i.e., $H(x, 0) \leq 0)$ (cf. 10$]$ ).

2.2. Main results. In order to prove the connection between 2.9 and a Beckmann-type problem, we will consider a slightly more general variant of $(2.9)$ by considering for $\rho \in \mathcal{M}_{b}(\Omega)$, the following maximization problem

$$
\left(\mathcal{M}_{D}\right): \max \left\{\int_{\Omega} u \mathrm{~d} \rho: u(y)-u(x) \leq d_{\sigma}(x, y), \forall x, y \in \Omega \text { and } u=g \text { on } D\right\} .
$$

Then, it is not difficult to see that the solution of $(2.9)$ can be recovered by taking $\rho \equiv 1$.

The connection with the Beckmann problem is given in the following theorem.

Theorem 2.3. The optimization problem $\left(\mathcal{M}_{D}\right)$ coincides with the following Beckmann-type problem

$$
(\mathcal{B K}): \quad \min _{\phi \in \mathcal{M}_{b}(\bar{\Omega})^{N}, \nu \in \mathcal{M}_{b}(D)}\left\{\int_{\bar{\Omega}} \sigma\left(x, \frac{\phi}{|\phi|}(x)\right) \mathrm{d}|\phi|+\int_{D} g \mathrm{~d} \nu:-\operatorname{div}(\phi)=\rho-\nu \text { in } \mathcal{D}^{\prime}\left(\mathbb{R}^{N}\right)\right\} .
$$

Moreover, $u$ and $(\phi, \nu)$ are optimal solutions to $\left(\mathcal{M}_{D}\right)$ and $(\mathcal{B K})$, respectively, if and only if

$$
\begin{cases}-\operatorname{div}(\phi)=\rho-\nu & \text { in } \mathcal{D}^{\prime}\left(\mathbb{R}^{N}\right) \\ \phi(x) \cdot \nabla_{|\phi|} u(x)=\sigma\left(x, \frac{\phi}{|\phi|}(x)\right) & |\phi|-\text { a.e. } x \\ u=g & \text { on } D,\end{cases}
$$

where $\nabla_{|\phi|} u$ denotes the tangential gradient with respect to $|\phi|$, the total variation of $\phi$ (cf. [4]).

In particular, we have the following.

Corollary 2.4. Let $u$ be the maximal viscosity subsolution to (2.8) and $(\phi, \nu)$ an optimal solution to $(\mathcal{B K})$ with $\rho=1$, then

$$
\begin{cases}-\operatorname{div}(\phi)=1-\nu & \text { in } \mathcal{D}^{\prime}\left(\mathbb{R}^{N}\right) \\ \phi(x) \cdot \nabla_{|\phi|} u(x)=\sigma\left(x, \frac{\phi}{|\phi|}(x)\right) & |\phi|-\text { a.e. } x \\ u=g & \text { on } D .\end{cases}
$$

For the case of non-degenerate Finsler metric $\sigma$ (i.e., $H(x, 0)<0)$ we know that, by $(1.5)$, the minimal value of $(\mathcal{B K})$ is the same with

$$
\left(\mathcal{M}_{N}\right): \min _{\nu \in \mathcal{M}_{b}(D)} \max _{u}\left\{\int_{\bar{\Omega}} u \mathrm{~d}(\rho-\nu)+\int_{D} g \mathrm{~d} \nu: u \text { is } 1-\text { Lipschitz w.r.t } d_{\sigma}\right\},
$$


as well as Monge-Kantorovich problem

$$
(\mathcal{M K}): \min _{\gamma \in \mathcal{M}^{+}(\bar{\Omega} \times \bar{\Omega}), \nu \in \mathcal{M}_{b}(D)}\left\{\int_{\bar{\Omega} \times \bar{\Omega}} d_{\sigma}(x, y) \mathrm{d} \gamma(x, y)+\int_{D} g \mathrm{~d} \nu: \pi_{1} \sharp \gamma=\rho^{-}+\nu^{+}, \pi_{2} \sharp \gamma=\rho^{+}+\nu^{-}\right\} .
$$

The following theorem ensures that the above-mentioned relations still hold true for the case of degenerate HJ equation, i.e., $H(x, 0) \leq 0$.

Theorem 2.5. Under the assumptions (H1-H3), we have

$$
\max \left(\mathcal{M}_{D}\right)=\min (\mathcal{B K})=\min \left(\mathcal{M}_{N}\right)=\min (\mathcal{M K}) .
$$

As a typical example we will consider an HJ equation of Eikonal type, coupled with a zero Dirichlet boundary condtion,

$$
\begin{cases}|\nabla u(x)|=k(x) & \text { in } \Omega \\ u=0 & \text { on } \partial \Omega .\end{cases}
$$

In other words, $H(x, p)=|p|-k(x)$ where $k$ is a continuous, nonnegative function on $\bar{\Omega}$. In this case, the problem $\left(\mathcal{M}_{D}\right)$ can be rewritten as

or

$$
\sup \left\{\int_{\Omega} u \mathrm{~d} x:|\nabla u| \leq k \text { and } u=0 \partial \Omega\right\}
$$

where $V=C^{1}(\Omega) \cap H_{0}^{1}(\Omega)$,

$$
(\mathcal{P}): \inf _{u \in V}\{\mathcal{F}(u)+\mathcal{G}(\Lambda u)\}
$$

$$
\mathcal{F}(u)=-\int_{\Omega} u \mathrm{~d} x, \quad \Lambda u=\nabla u \quad \text { and } \quad \mathcal{G}(q)= \begin{cases}0 & \text { if }|q| \leq k \\ +\infty & \text { otherwise }\end{cases}
$$

For the case of non-degeneracy, i.e., $k(x)>0$ on $\bar{\Omega}$, the Fenchel-Rockafellar duality, since the qualification conditions are satisfied (see e.g. [11, Theorem III 4.1]), gives

$$
\sup \left\{\int_{\Omega} u \mathrm{~d} x:|\nabla u| \leq k \text { and } u=0 \text { on } \partial \Omega\right\}=\min _{\phi \in \mathcal{M}_{b}(\bar{\Omega})^{N}}\left\{\int_{\bar{\Omega}} k \mathrm{~d}|\phi|:-\operatorname{div}(\phi)=\rho \text { in } \mathcal{D}^{\prime}(\Omega)\right\} .
$$

However, dealing with general degenerate Hamiltionians at least two difficulties arise. Firstly, the qualification conditions are not satisfied to apply directly the Fenchel-Rockafellar duality. Secondly, in this setting the problem $(\mathcal{B K})$ is not coercive, it follows that the existence of an optimal solution to $(\mathcal{B K})$ is not trivial. These two issues will be addressed in this paper via approximation and optimal transport techniques.

Before ending this section, let us mention that thanks to the duality result in [10], we have the following

Corollary 2.6. The extremal values $(\mathcal{B K})$ and

$$
\left(\mathcal{O F}_{D}\right): \inf _{\phi \in \mathcal{D M}^{p}(\Omega)}\left\{\int_{\Omega} \sigma(x, \phi(x)) \mathrm{d} x-\langle\phi \cdot \mathbf{n}, g\rangle:-\operatorname{div}(\phi)=\rho \operatorname{in} \mathcal{D}^{\prime}(\Omega)\right\},
$$

coincide. 
The formulation of the problem $\left(\mathcal{O F}_{D}\right)$ as well as the definition of $\mathcal{D} \mathcal{M}^{p}(\Omega)$ and further comments are recalled in Remark 3.10 .

\section{Proofs}

3.1. Preparatory results. Let $\nu \in \mathcal{M}_{b}(D)$ satisfy $\rho(\Omega)=\nu(D)$. Define two functionals

$$
\begin{aligned}
\mathcal{T}: L^{1}(\Omega)^{N} \mapsto \mathbb{R} \cup\{+\infty\}, & \phi \mapsto \mathcal{T}(\phi)= \begin{cases}\int_{\Omega} \sigma(x, \phi(x)) \mathrm{d} x & \text { if }-\operatorname{div}(\phi)=\rho-\nu \text { in } \mathcal{D}^{\prime}\left(\mathbb{R}^{N}\right) \\
+\infty & \text { otherwise, }\end{cases} \\
\mathcal{E}: \operatorname{Lip}(\Omega) \mapsto \mathbb{R} \cup\{-\infty\}, & u \mapsto \mathcal{E}(u)= \begin{cases}\int_{\bar{\Omega}} u \mathrm{~d}(\rho-\nu) & \text { if } \sigma^{*}(x, \nabla u(x)) \leq 1 \text { a.e. } x \in \Omega \\
-\infty & \text { otherwise. }\end{cases}
\end{aligned}
$$

Lemma 3.7. Assume that $\sigma$ is a degenerate Finsler metric. Let $\mathcal{T}, \mathcal{E}$ be defined as above and $\rho(\Omega)=\nu(D)$. Then

$$
\inf _{\phi \in L^{1}(\Omega)^{N}} \mathcal{T}(\phi)=\sup _{u \in \operatorname{Lip}(\Omega)} \mathcal{E}(u)
$$

Proof. The proof will be divided into two steps. We first prove for the case of non-degenerate Finsler metric $\sigma$, i.e., there exist two positive constants $K_{1}, K_{2}$ such that

$$
K_{1}|p| \leq \sigma(x, p) \leq K_{2}|p| \text { for any } x \in \bar{\Omega}, p \in \mathbb{R}^{N} .
$$

In this setting, due to the non-degeneracy of $\sigma$, the qualification conditions are satisfied and the result follows directly from the Fenchel-Rockafellar duality (see e.g. [11, Theorem III 4.1]). For the general case, we check at once that $\sup \mathcal{E} \leq \inf \mathcal{T}$ by taking $u$ as a test function in the divergence constraint $-\operatorname{div}(\phi)=\rho-\nu$ in $\mathcal{D}^{\prime}\left(\mathbb{R}^{N}\right)$. Therefore, it remains to prove that

$$
\inf \mathcal{T} \leq \sup \mathcal{E}
$$

We now proceed by an approximation via non-degenerate Finsler metrics. For $n \in \mathbb{N}^{*}$ and $x \in \bar{\Omega}$, define

$$
\sigma_{n}(x, p):=\max \left(\sigma(x, p), \frac{|p|}{n}\right) \text { for every } p \in \mathbb{R}^{N},
$$

which establishes a sequence of non-degenerate Finsler metrics $\sigma_{n}$ on $\bar{\Omega}$ satisfying

$$
\frac{|p|}{n} \leq \sigma_{n}(x, p) \leq K|p| \text { for some constant } K>0 .
$$

Thanks to [7, Thereom 5.1], we have that $d_{\sigma_{n}} \rightarrow d_{\sigma}$ in the space of Finsler distances endowed with the topology induced by uniform convergence on compact subsets of $\bar{\Omega} \times \bar{\Omega}$. To prove the 
inverse inequality (3.10), let us introduce for $n \in \mathbb{N}^{*}$ the functionals

$$
\begin{aligned}
\mathcal{T}_{n}: L^{1}(\Omega)^{N} \mapsto \mathbb{R} \cup\{+\infty\}, & \phi \begin{cases}\int_{\Omega} \sigma_{n}(x, \phi(x)) \mathrm{d} x & \text { if }-\operatorname{div}(\phi)=\rho-\nu \text { in } \mathcal{D}^{\prime}\left(\mathbb{R}^{N}\right) \\
+\infty & \text { otherwise, }\end{cases} \\
\mathcal{E}_{n}: \operatorname{Lip}(\Omega) \mapsto \mathbb{R} \cup\{-\infty\}, & u \mapsto \begin{cases}\int_{\bar{\Omega}} u \mathrm{~d}(\rho-\nu) & \text { if } \sigma_{n}^{*}(x, \nabla u(x)) \leq 1 \text { a.e. } x \in \Omega \\
-\infty & \text { otherwise. }\end{cases}
\end{aligned}
$$

It follows from the non-degeneracy of $\sigma_{n}$ and the first step of the proof that inf $\mathcal{T}_{n}=\sup \mathcal{E}_{n}$. We are now in a position to show that $\sup \mathcal{E}_{n} \rightarrow \sup \mathcal{E}$ as $n \rightarrow \infty$. Let $u_{n}$ be a maximizer for $\mathcal{E}_{n}$, i.e., $\sup \mathcal{E}_{n}=\int_{\bar{\Omega}} u_{n} \mathrm{~d}(\rho-\nu)$ and $\sigma_{n}^{*}\left(x, \nabla u_{n}(x)\right) \leq 1$ a.e. in $\Omega$. Fix $x_{0} \in \Omega$. Since $\rho(\Omega)=\nu(D)$, we see that $u_{n}:=u_{n}-u_{n}\left(x_{0}\right)$ is still a maximizer. Thus we can assume that $u_{n}\left(x_{0}\right)=0$ for any $n$. Since

$$
u_{n}(y)-u_{n}(x) \leq d_{\sigma_{n}}(x, y) \leq K|x-y| \text { in } \Omega \times \Omega,
$$

$\left\{u_{n}\right\}$ is equi-Lipschitz continuous. By Ascoli-Arzelà's theorem, there exists a Lipschitz function $u$ such that, up to a subsequence, $u_{n} \rightrightarrows u$ uniformly in $\Omega$. Since $d_{\sigma_{n}} \rightarrow d_{\sigma}$ as $n \rightarrow \infty$ we deduce that $u$ is admissible for $\mathcal{E}$, i.e. $u(y)-u(x) \leq d_{\sigma}(x, y)$ in $\Omega \times \Omega$. Consequently

$$
\lim _{n}\left(\sup \mathcal{E}_{n}\right)=\lim _{n} \int_{\bar{\Omega}} u_{n} \mathrm{~d}(\rho-\nu)=\int_{\bar{\Omega}} u \mathrm{~d}(\rho-\nu) \leq \sup \mathcal{E}
$$

and

$$
\inf \mathcal{T} \leq \lim _{n}\left(\inf \mathcal{T}_{n}\right) \leq \sup \mathcal{E}
$$

as claimed in 3.10 .

Before ending up this subsection, we recall the notion of disintegration of measures which will be useful in the proof of existence of optimal solution to the Beckmann-type problem.

Theorem 3.8. (cf. [21]) Let $X, Y$ be locally compact metric spaces and $\pi: X \rightarrow Y$ a Borel map. For any $\eta \in \mathcal{M}_{b}^{+}(X)$ there exist a family of probability measures $\left(\eta_{y}\right)_{y \in Y}$ on $X$ concentrated on $\pi^{-1}(\{y\})$ such that for any test function $u \in C(X)$, the mapping $y \mapsto \int_{X} u \mathrm{~d} \eta_{y}$ is Borel measurable and

$$
\int_{X} u(x) \mathrm{d} \eta(x)=\int_{Y} \int_{X} u(x) \mathrm{d} \eta_{y}(x) \mathrm{d} \pi_{\sharp} \eta(y) .
$$

3.2. Proofs of the main results. We get started with the proof of Theorem 2.3 by the following result.

Proposition 3.9. We have

$$
\max \left(\mathcal{M}_{D}\right)=\inf (\mathcal{B K})=\inf \widetilde{(\mathcal{B K})}
$$


where

$$
\widetilde{(\mathcal{B K})}: \inf _{\phi \in L^{1}(\Omega)^{N}, \nu \in \mathcal{M}_{b}(D)}\left\{\int_{\Omega} \sigma(x, \phi(x)) \mathrm{d} x+\int_{D} g \mathrm{~d} \nu:-\operatorname{div}(\phi)=\rho-\nu \text { in } \mathcal{D}^{\prime}\left(\mathbb{R}^{N}\right)\right\} .
$$

Proof. First observe that

$$
\max \left(\mathcal{M}_{D}\right) \leq \inf (\mathcal{B K}) \leq \inf \widetilde{(\mathcal{B K})}
$$

Indeed, take $u$ satisfying $\sigma^{*}(x, \nabla u(x)) \leq 1$ a.e. $x$ in $\Omega, u=g$ on $D$ as a test function in the divergence constraint $-\operatorname{div}(\phi)=\rho-\nu$ in $\mathcal{D}^{\prime}\left(\mathbb{R}^{N}\right)$, we get

$$
\int_{\Omega} u \mathrm{~d} \rho=\int_{\Omega} \frac{\phi}{|\phi|} \cdot \nabla u \mathrm{~d}|\phi|+\int_{D} g \mathrm{~d} \nu \leq \int_{\Omega} \sigma\left(x, \frac{\phi}{|\phi|}(x)\right) \mathrm{d}|\phi|+\int_{D} g \mathrm{~d} \nu .
$$

This implies (3.11).

So, it is sufficient to show the duality between $\left(\mathcal{M}_{D}\right)$ and $\widetilde{(\mathcal{B K})}$, i.e. $\max \left(\mathcal{M}_{D}\right)=\inf \widetilde{(\mathcal{B K})}$. We use a perturbation technique as in [8]. Define on $\mathcal{C}(D)$ the following functional

$$
F: v \in \mathcal{C}(D) \mapsto-\max _{u}\left\{\int_{\Omega} u \mathrm{~d} \rho: u \in \operatorname{Lip}(\Omega), \sigma^{*}(x, \nabla u(x)) \leq 1, u+v=g \text { on } D\right\},
$$

which is well-defined. Let us show that $F$ is convex and l.s.c.. Consider $v_{1}, v_{2} \in \mathcal{C}(D)$ and set $v=t v_{1}+(1-t) v_{2}$ for $t \in[0,1]$. Let $u_{1}, u_{2} \in \operatorname{Lip}(\Omega)$ be two maximizers corresponding to $v_{1}$ and $v_{2}$ respectively, i.e. $\sigma^{*}\left(x, \nabla u_{i}(x)\right) \leq 1, u_{i}+v_{i}=g$ on $D$ and

$$
F\left(v_{i}\right)=-\int_{\Omega} u_{i} \mathrm{~d} \rho \text { for } i=1,2 .
$$

Define $u=t u_{1}+(1-t) u_{2}$. It is evident that $u+v=g$ on $D$. And using the homogeneity of $\sigma^{*}$, we obtain $\sigma^{*}(x, \nabla u) \leq t \sigma^{*}\left(x, \nabla u_{1}\right)+(1-t) \sigma^{*}\left(x, \nabla u_{2}\right) \leq 1$ so that $u$ is admissible for $v$. Finally, we get

$$
F(v) \leq-\int_{\Omega} u \mathrm{~d} \rho=t\left(-\int_{\Omega} u_{1} \mathrm{~d} \rho\right)+(1-t)\left(-\int_{\Omega} u_{2} \mathrm{~d} \rho\right)=t F\left(v_{1}\right)+(1-t) F\left(v_{2}\right),
$$

which proves the convexity. For the lower semicontinuity, take a sequence $v_{n} \rightrightarrows v$ uniformly on $D$. For every $n \in \mathbb{N}$, consider a maximizer $u_{n}$ corresponding to $v_{n}$ such that $u_{n}+v_{n}=g$ on $D$, $u_{n}$ are 1 -Lipschitz w.r.t. $d_{\sigma}$ (i.e., $u_{n}(y)-u_{n}(x) \leq d_{\sigma}(x, y)$ or equivalently, $\sigma^{*}\left(x, \nabla u_{n}(x)\right) \leq 1$ a.e. $x \in \Omega$ ), and

$$
F\left(v_{n}\right)=-\int_{\Omega} u_{n} \mathrm{~d} \rho .
$$

Since $u_{n}(y)-u_{n}(x) \leq d_{\sigma}(x, y) \leq K|x-y|$, the functions $u_{n}$ are equi-Lipschitz in the Euclidean distance. Since the sequence $\left\{v_{n}\right\}$ is convergent, it is bounded on $D$, and so is the sequence $\left\{u_{n}\right\}$. By Ascoli-Arzelà's theorem, there exists a Lipschitz function $u$ such that $u_{n} \rightrightarrows u$ uniformly in $\Omega$ as $n \rightarrow \infty$. It is clear that $u+v=g$ on $D$ and $u(y)-u(x) \leq d_{\sigma}(x, y)$, i.e. $u$ is admissible for $v$. The lower semicontinuity is completed by

$$
F(v) \leq-\int_{\Omega} u \mathrm{~d} \rho=\lim _{n \rightarrow \infty}-\int_{\Omega} u_{n} \mathrm{~d} \rho=\liminf _{n \rightarrow \infty} F\left(v_{n}\right) .
$$


Since $F$ is convex and l.s.c., we have $F=F^{* *}$, in particular $F(0)=F^{* *}(0)$. Let us finish the proof by computing $F^{*}$ and $F^{* *}$. For any $\nu \in \mathcal{M}_{b}(D)$, we see that

$$
\begin{aligned}
F^{*}(\nu) & =\sup _{v \in \mathcal{C}(D)} \int_{D} v \mathrm{~d} \nu-F(v) \\
& =\sup _{v \in \mathcal{C}(D), u \in \operatorname{Lip}(\Omega)}\left\{\int_{D} v \mathrm{~d} \nu+\int_{\Omega} u \mathrm{~d} \rho: \sigma^{*}(x, \nabla u(x)) \leq 1, u+v=g \text { on } D\right\} \\
& \left.=\sup _{u \in \operatorname{Lip}(\Omega)}\left\{\int_{\Omega} u \mathrm{~d} \rho+\int_{D}(g-u) \mathrm{d} \nu: \sigma^{*}(x, \nabla u(x))\right) \leq 1, g-u \in \mathcal{C}(D)\right\} .
\end{aligned}
$$

For any constant $c \in \mathbb{R}$ and a Lipschitz extension $\tilde{g}$ of $g$, one can see that $u:=\tilde{g}+c$ is an admissible test function in the definition of $F^{*}(\nu)$ and

$$
\int_{\Omega} u \mathrm{~d} \rho+\int_{D}(g-u) \mathrm{d} \nu=c(\rho(\Omega)-\nu(D))+\int_{\Omega} \tilde{g} \mathrm{~d} \rho .
$$

So, if $\rho(\Omega) \neq \nu(D)$, then $F^{*}(\nu)=+\infty$. This implies that

$$
F^{*}(\nu)= \begin{cases}\left.\sup _{u \in \operatorname{Lip}(\Omega)}\left\{\int_{\Omega} u \mathrm{~d} \rho+\int_{D}(g-u) \mathrm{d} \nu: \sigma^{*}(x, \nabla u(x))\right) \leq 1\right\} & \text { if } \nu(D)=\rho(\Omega) \\ +\infty & \text { otherwise. }\end{cases}
$$

Following Lemma 3.7, for any $\nu \in \mathcal{M}_{b}(D)$ such that $\nu(D)=\rho(\Omega)$, we have

$$
\begin{aligned}
F^{*}(\nu) & \left.=\sup _{u \in \operatorname{Lip}(\Omega)}\left\{\int_{\Omega} u \mathrm{~d} \rho+\int_{D}(g-u) \mathrm{d} \nu: \sigma^{*}(x, \nabla u(x))\right) \leq 1\right\} \\
& \left.=\int_{D} g \mathrm{~d} \nu+\sup _{u \in \operatorname{Lip}(\Omega)}\left\{\int_{\bar{\Omega}} u \mathrm{~d}(\rho-\nu): \sigma^{*}(x, \nabla u(x))\right) \leq 1\right\} \\
& =\int_{D} g \mathrm{~d} \nu+\inf _{\phi \in L^{1}(\Omega)^{N}}\left\{\int_{\Omega} \sigma(x, \phi(x)) \mathrm{d} x:-\operatorname{div}(\phi)=\rho-\nu \operatorname{in} \mathcal{D}^{\prime}\left(\mathbb{R}^{N}\right)\right\} \\
& =\inf _{\phi \in L^{1}(\Omega)^{N}}\left\{\int_{\Omega} \sigma(x, \phi(x)) \mathrm{d} x+\int_{D} g \mathrm{~d} \nu:-\operatorname{div}(\phi)=\rho-\nu \operatorname{in} \mathcal{D}^{\prime}\left(\mathbb{R}^{N}\right)\right\} .
\end{aligned}
$$

Consequently,

$$
\max \left(\mathcal{M}_{D}\right)=-F(0)=-F^{* *}(0)=-\sup _{\nu \in \mathcal{M}_{b}(D)}-F^{*}(\nu)=\inf \widetilde{(\mathcal{B K})}
$$

Remark 3.10. Following our approach in [10], it is possible to show that the optimal $\nu$ in $(\mathcal{B K})$ is somehow related to the trace of the optimal flow $\phi$. For completeness let us recall briefly some of 
the main ingredients we used, and for a simpler presentation, consider the case where $D=\partial \Omega$. Then we prove in [10] the duality between $\left(\mathcal{M}_{D}\right)$ and

$$
\left(\mathcal{O F}_{D}\right): \inf _{\phi \in \mathcal{D} \mathcal{M}^{p}(\Omega)}\left\{\int_{\Omega} \sigma(x, \phi(x)) d x-\langle\phi \cdot \mathbf{n}, g\rangle:-\operatorname{div}(\phi)=\rho \operatorname{in} \mathcal{D}^{\prime}(\Omega)\right\},
$$

where we define for $1 \leq p \leq \infty$,

$$
\mathcal{D} \mathcal{M}^{p}(\Omega):=\left\{\phi \in L^{p}(\Omega)^{N}: \operatorname{div} \phi=: \mu \in \mathcal{M}_{b}(\Omega)\right\},
$$

endowed with the graph norm

$$
\|\phi\|_{\mathcal{D} \mathcal{M}^{p}(\Omega)}:=\|\phi\|_{L^{p}(\Omega)}+|\operatorname{div} \phi|(\Omega) .
$$

The main interest of introducing such a space is to give a sense to the trace term $\phi \cdot \mathbf{n}$ which is not always defined for a general measure field $\phi$. In particular, for any measure field $\phi \in \mathcal{D} \mathcal{M}^{p}(\Omega)$, one can define a trace $\phi \cdot \mathbf{n}$ on $\partial \Omega$ as a linear form on $\operatorname{Lip}(\partial \Omega)$ such that

$$
\left\langle\phi \cdot \mathbf{n}, \xi_{/ \partial \Omega}\right\rangle=\int_{\Omega} \xi \operatorname{div} \phi+\int_{\Omega} \nabla \xi \cdot \phi, \quad \text { for any } \xi \in \mathcal{C}^{1}(\bar{\Omega}) .
$$

One can see that, at least formally, $\nu$ plays the role of $-\phi \cdot \mathbf{n}$ in $\left(\mathcal{O F} \mathcal{F}_{D}\right)$.

Our aim now is to use the optimal mass transportation interpretation to prove that the inf in $(\mathcal{B K})$ is actually a min, i.e. the existence of optimal solution to the Beckmann-type problem $(\mathcal{B K})$.

Proposition 3.11. There exist $\nu \in \mathcal{M}_{b}(D)$ and a vector measure $\Phi$ such that $-\operatorname{div}(\Phi)=\rho-\nu$ in $\mathcal{D}^{\prime}\left(\mathbb{R}^{N}\right)$ and

$$
\inf (\mathcal{B K}) \leq \int_{\bar{\Omega}} \sigma\left(x, \frac{\Phi}{|\Phi|}\right) \mathrm{d}|\Phi|+\int_{D} g \mathrm{~d} \nu \leq \min (\mathcal{M K}) .
$$

In particular we see that $\min (\mathcal{B K})=\max \left(\mathcal{M}_{D}\right)$.

Proof. Take $(\gamma, \nu)$ be a solution of $(\mathcal{M K})$ and define a vector measure $\Phi_{\gamma}$ through

$$
<\Phi_{\gamma}, v>=\int_{\bar{\Omega} \times \bar{\Omega}} \int_{0}^{1} v(\xi(t)) \dot{\xi}(t) \mathrm{d} t \mathrm{~d} \gamma(x, y) \quad \forall v \in \mathcal{C}(\bar{\Omega})^{N}
$$

with $\xi$ being a geodesic joining $x$ and $y$ with respect to $d_{\sigma}$. Let us check the feasibility of $\Phi_{\gamma}$ for $(\mathcal{B K})$, i.e.

$$
-\operatorname{div}\left(\Phi_{\gamma}\right)=\rho-\nu \operatorname{in} \mathcal{D}^{\prime}\left(\mathbb{R}^{N}\right)
$$

For any $w \in \mathcal{C}^{1}(\bar{\Omega})$, by definition, we have

$$
\begin{aligned}
<\Phi_{\gamma}, \nabla w> & =\int_{\Omega \times \Omega} \int_{0}^{1} \frac{d w(\xi(t))}{d t} \mathrm{~d} t \mathrm{~d} \gamma(x, y) \\
& =\int_{\Omega \times \Omega}(w(y)-w(x)) \mathrm{d} \gamma(x, y) \\
& =\int_{\bar{\Omega}} w \mathrm{~d}(\rho-\nu),
\end{aligned}
$$


which gives $(3.12)$. The next task is to show that

$$
\int_{\bar{\Omega}} \sigma\left(x, \frac{\Phi_{\gamma}}{\left|\Phi_{\gamma}\right|}(x)\right) \mathrm{d}\left|\Phi_{\gamma}\right|+\int_{D} g \mathrm{~d} \nu \leq \int_{\bar{\Omega} \times \bar{\Omega}} d_{\sigma}(x, y) \mathrm{d} \gamma(x, y)+\int_{D} g \mathrm{~d} \nu .
$$

To do so, for each $t \in[0,1]$, define vector measure $E_{t}$ by setting $E_{t}(v):=\int_{\bar{\Omega} \times \bar{\Omega}} v(\xi(t)) \dot{\xi}(t) \mathrm{d} \gamma(x, y)$ for $v \in \mathcal{C}(\bar{\Omega})^{N}$. We get $\Phi_{\gamma}=\int_{0}^{1} E_{t} \mathrm{~d} t$ and, by Jensen's inequality,

$$
\int_{\bar{\Omega}} \sigma\left(x, \frac{\Phi_{\gamma}}{\left|\Phi_{\gamma}\right|}(x)\right) \mathrm{d}\left|\Phi_{\gamma}\right| \leq \int_{0}^{1} \int_{\bar{\Omega}} \sigma\left(x, \frac{E_{t}}{\left|E_{t}\right|}(x)\right) \mathrm{d}\left|E_{t}\right| \mathrm{d} t .
$$

Now define $\pi_{t}:(x, y) \in \bar{\Omega} \times \bar{\Omega} \mapsto \xi(t)$ for $t \in[0,1]$, where $\xi$ is as before, a geodesic joining $x$ and $y$. Consider $\eta_{t}=\left(\pi_{t}\right)_{\sharp} \gamma$. Using disintegration theorem (see Theorem 3.8) for $\gamma$ with respect to $\pi_{t}$, we can find probability measures $\gamma_{z}$ supported on $\pi_{t}^{-1}(\{z\})$ such that

$$
\int_{\bar{\Omega} \times \bar{\Omega}} u(x, y) \mathrm{d} \gamma(x, y)=\int_{\bar{\Omega} \times \bar{\Omega} \times \bar{\Omega}} u(x, y) \mathrm{d} \gamma_{z}(x, y) \mathrm{d} \eta_{t}(z) .
$$

We check at once that $E_{t}<<\eta_{t}$ with the density $\frac{d E_{t}}{d \eta_{t}}(z)=\int_{\bar{\Omega} \times \bar{\Omega}} \dot{\xi}(t) \mathrm{d} \gamma_{z}(x, y)$, which follows from the fact that, for any test function $v$,

$$
\begin{aligned}
\int_{\bar{\Omega}} v(z) \mathrm{d} E_{t} & =\int_{\bar{\Omega}} v(\xi(t)) \dot{\xi}(t) \mathrm{d} \gamma(x, y) \\
& =\int_{\bar{\Omega} \times \bar{\Omega} \times \bar{\Omega}} v(\xi(t)) \dot{\xi}(t) \mathrm{d} \gamma_{z}(x, y) \mathrm{d} \eta_{t}(z) \\
& =\int_{\bar{\Omega}} v(z) \int_{\bar{\Omega} \times \bar{\Omega}} \dot{\xi}(t) \mathrm{d} \gamma_{z}(x, y) \mathrm{d} \eta_{t}(z) .
\end{aligned}
$$

On the other hand, by 3.14 and Jensen's inequality,

$$
\begin{aligned}
\int_{\bar{\Omega} \times \bar{\Omega}} \sigma(\xi(t), \dot{\xi}(t)) \mathrm{d} \gamma(x, y) & =\int_{\bar{\Omega} \times \bar{\Omega} \times \bar{\Omega}} \sigma(\xi(t), \dot{\xi}(t)) \mathrm{d} \gamma_{z}(x, y) \mathrm{d} \eta_{t}(z) \\
& =\int_{\bar{\Omega} \times \bar{\Omega} \times \bar{\Omega}} \sigma(z, \dot{\xi}(t)) \mathrm{d} \gamma_{z}(x, y) \mathrm{d} \eta_{t}(z) \\
& \geq \int_{\bar{\Omega}} \sigma\left(z, \int_{\bar{\Omega} \times \bar{\Omega}} \dot{\xi}(t) \mathrm{d} \gamma_{z}(x, y)\right) \mathrm{d} \eta_{t}(z) \\
& =\int_{\bar{\Omega}} \sigma\left(z, \frac{E_{t}}{\left|E_{t}\right|}(z)\right) \mathrm{d}\left|E_{t}\right|(z) .
\end{aligned}
$$

Finally, we observe that since $\xi$ is a geodesic

$$
\int_{\bar{\Omega} \times \bar{\Omega}} d_{\sigma}(x, y) \mathrm{d} \gamma(x, y)+\int_{D} g \mathrm{~d} \nu=\int_{\bar{\Omega} \times \bar{\Omega}} \int_{0}^{1} \sigma(\xi(t), \dot{\xi}(t)) \mathrm{d} \gamma(x, y) d t+\int_{D} g \mathrm{~d} \nu
$$




$$
\begin{aligned}
& \geq \int_{0}^{1} \int_{\bar{\Omega}} \sigma\left(z, \frac{E_{t}}{\left|E_{t}\right|}(z)\right) \mathrm{d}\left|E_{t}\right|(z)+\int_{D} g \mathrm{~d} \nu(\text { by } 3.15) \\
& \left.\geq \int_{\bar{\Omega}} \sigma\left(x, \frac{\Phi_{\gamma}}{\left|\Phi_{\gamma}\right|}(x)\right) \mathrm{d}\left|\Phi_{\gamma}\right|+\int_{D} g \mathrm{~d} \nu \text { (by } 3.13\right) .
\end{aligned}
$$

Consequently

$$
\inf (\mathcal{B K}) \leq \int_{\bar{\Omega}} \sigma\left(x, \frac{\Phi_{\gamma}}{\left|\Phi_{\gamma}\right|}(x)\right) \mathrm{d}\left|\Phi_{\gamma}\right|+\int_{D} g \mathrm{~d} \nu \leq \int_{\bar{\Omega} \times \bar{\Omega}} d_{\sigma}(x, y) \mathrm{d} \gamma(x, y)+\int_{D} g \mathrm{~d} \nu=\min (\mathcal{M K}) .
$$

Proof of Theorem 2.5. Following Theorem 1.1 on Kantorovich duality, one has $\max \left(\mathcal{M}_{N}\right)=$ $\min (\mathcal{M K})$. Combining this with Propositions 3.9 and 3.11, we conclude that the Beckmann-type problem $(\mathcal{B K})$ admits an optimal solution and $\max \left(\mathcal{M}_{D}\right)=\min (\mathcal{B K})=\max \left(\mathcal{M}_{N}\right)=\min (\mathcal{M K})$.

Proof of Theorem 2.3. The proof of the duality between $\max \left(\mathcal{M}_{D}\right)$ and $\inf (\mathcal{B K})$ is followed from Proposition 3.9 while the existence of optimal solution to the Beckmann-type problem $(\mathcal{B K})$ is a consequence of Proposition 3.11 (see also Theorem 2.5). Let us now show the optimality conditions. Indeed, $u$ and $(\phi, \nu)$ are optimal solutions for $\left(\mathcal{M}_{D}\right)$ and $(\mathcal{B K})$, respectively, if and only if

$$
\int_{\Omega} \sigma\left(x, \frac{\phi}{|\phi|}(x)\right) \mathrm{d}|\phi|+\int_{D} g \mathrm{~d} \nu=\int_{\Omega} u \mathrm{~d} \rho
$$

or

$$
\int_{\Omega} \sigma\left(x, \frac{\phi}{|\phi|}(x)\right) \mathrm{d}|\phi|=\int_{\bar{\Omega}} u \mathrm{~d}(\rho-\nu)=\int_{\Omega} \phi(x) \cdot \nabla_{|\phi|} u(x) \mathrm{d}|\phi| .
$$

This is equivalent to

$$
\sigma\left(x, \frac{\phi}{|\phi|}(x)\right)=\phi(x) \cdot \nabla_{|\phi|} u(x) \quad \text { for }|\phi| \text {-a.e. } x,
$$

as desired.

\section{Numerical Results}

As we pointed out in the previous sections, the maximization problem $\left(\mathcal{M}_{D}\right)$ is linked to the Monge-Kantorovich type problem $(\mathcal{M K})$. The measure $\rho$ needs not to satisfy the standard mass balance condition. However, transporting a part of the mass from/to the Dirichlet region $D$ is allowed. In addition, taking $\rho=\mathcal{L}_{\mid \Omega}^{N}$ the restriction of Lebesgue measure on $\Omega,\left(\mathcal{M}_{D}\right)$ allows recovering the solution of HJ equation. This was essentially addressed in [10] for several Hamiltonians. Here, we will focus essentially on the solution of $(\mathcal{B K})$. 
4.1. Formulation of the problem. We set $\mathscr{X}=W^{1, \infty}(\Omega)$ and $\mathscr{Y}=L^{\infty}(\Omega)^{N} \times \mathcal{C}(D)$, with

$$
\mathcal{F}(u)=-\int_{\bar{\Omega}} u \mathrm{~d} \rho, \quad \Lambda u=\left(\nabla u, u_{\mid D}\right), \quad \text { for any } u \in \mathscr{X}
$$

and

$$
G(\eta, h)=\left\{\begin{array}{ll}
0 & \text { if } \sigma^{*}(x, \eta) \leq 1 \text { and } h=g \text { on } D \\
+\infty & \text { otherwise, }
\end{array} \text { for any }(\eta, h) \in \mathscr{Y} .\right.
$$

Thus, we can rewrite the problem $\left(\mathcal{M}_{D}\right)$ in the form

$$
-\inf _{u \in \mathscr{X}} F(u)+\mathcal{G}(\Lambda u) .
$$

Thanks to Theorem 2.5) and Proposition (3.9), we have

$$
\begin{gathered}
-\min \{\mathcal{F}(u)+\mathcal{G}(\Lambda u): u \in \mathscr{X}\}=-\sup \left\{-\mathcal{F}^{*}\left(-\Lambda^{*}(\phi, \nu)\right)-\mathcal{G}^{*}(\phi, \nu): \phi \in L^{1}(\Omega)^{N}, \nu \in \mathcal{M}_{b}(D)\right\} \\
=\min _{\phi \in L^{1}(\Omega)^{N}, \nu \in \mathcal{M}_{b}(D)}\left\{\int_{\Omega} \sigma(x, \phi(x)) \mathrm{d} x+\int_{D} g \mathrm{~d} \nu:-\operatorname{div}(\phi)=\rho-\nu \operatorname{in} \mathcal{D}^{\prime}\left(\mathbb{R}^{N}\right)\right\} .
\end{gathered}
$$

Introducing a new primal variable $(p, q) \in \mathscr{Y},\left(\mathcal{M}_{D}\right)$ reads

$$
-\inf _{\substack{(u,(p, q)) \in \mathscr{X} \times \mathscr{Y} \\ \Lambda u=(p, q)}} \mathcal{F}(u)+\mathcal{G}(p, q) .
$$

This allows us to rewrite $\left(\mathcal{M}_{D}\right)$ and $(\mathcal{B K})$ in a saddle point form

$$
(\mathscr{S}): \min _{(u,(p, q)) \in \mathscr{X} \times \mathscr{Y}} \sup _{\phi \in L^{1}(\Omega)^{N}, \nu \in \mathcal{M}_{b}(D)} L(u,(p, q) ; \phi, \nu)
$$

where

$$
L(u,(p, q) ; \phi, \nu)=\mathcal{F}(u)+\mathcal{G}(p, q)+\int_{\Omega} \phi \cdot(\nabla u-p) \mathrm{d} x+\int_{D} \nu \cdot(u-q) \mathrm{d} x .
$$

As usual, it is convenient to consider the following augmented Lagrangian

$$
L_{r}(u,(p, q) ; \phi, \nu)=L(u,(p, q) ; \phi, \nu)+\frac{r}{2}|\nabla u-p|^{2}+\frac{r}{2}\left|u_{\mid D}-q\right|^{2}, r>0,
$$

which has the same saddle points as $L$. Thus, the problem we will focus on is

$$
\left(\mathscr{S}_{r}\right): \min _{(u, q) \in \mathscr{X} \times \mathscr{Y}} \sup _{\phi \in L^{1}(\Omega)^{N}, \nu \in \mathcal{M}_{b}(D)} L_{r}(u,(p, q) ; \phi, \nu) .
$$

The numerical approximation of $\left(\mathcal{M}_{D}\right)$ was addressed in [10]. The main difference with the above formulation and the one in [10] is the presence of an additional Lagrange multiplier $\nu$ corresponding to the boundary condition $u=g$ on $D$. The resolution of $\left(\mathcal{M}_{D}\right)$ in [10] was used via ALG2 algorithm [11, 15] and was implemented using finite element method. Up to our knowledge, it is not straightforward to define finite element functions $\nu$ on a closed subset $D$ (typically $D=\partial \Omega$ ). In the next section we explain how to tackle this difficulty. 
4.2. Practical implementation. As we pointed out in the previous subsection, it is not clear how to solve the saddle point problem $\left(\mathscr{S}_{r}\right)$ via ALG2 algorithm due to the presence of the measure $\nu$ on the Dirichlet region. However, thanks to Corollary 2.6 and Remark 3.10 which shows that $\nu$ is somehow linked to the trace of the optimal flow $\phi$ on $D$, we can restrict ourselves to a formulation involving only the potential $u$ and the flow $\phi$. More precisely, for any $u \in \mathscr{X}$ and $\eta \in Z=L^{\infty}(\Omega)$, we take

$\mathcal{F}(u)=\left\{\begin{array}{ll}-\int_{\Omega} u \mathrm{~d} \rho & \text { if } u=g \text { on } D \\ +\infty & \text { otherwise }\end{array}, \quad \mathcal{G}(\eta)=\left\{\begin{array}{ll}0 & \text { if } \sigma^{*}(x, \eta) \leq 1 \\ +\infty & \text { otherwise }\end{array}, \quad\right.\right.$ and $\Lambda u=\nabla u$

Thus, following our approach in [10], we can focus on the following saddle point problem

$$
\inf _{(u, q) \in \mathscr{X} \times Z} \sup _{\phi \in \mathcal{D} \mathcal{M}^{p}(\Omega \backslash D)} L(u, q ; \phi)
$$

where

$$
L(u, q ; \phi)=\mathcal{F}(u)+\mathcal{G}(q)+\int_{\Omega} \phi \cdot(\Lambda(u)-q) d x, \quad \text { for any }(u, q, \phi) \in \mathscr{X} \times Z \times \mathcal{D} \mathcal{M}^{p}(\Omega \backslash D)
$$

Hence, the augmented Lagrangian reads

$L_{r}(u, q ; \phi)=\mathcal{F}(u)+\mathcal{G}(q)+\langle\phi, \Lambda u-q\rangle+\frac{r}{2}|\Lambda u-q|^{2}, \quad$ for any $(u, q, \phi) \in \mathscr{X} \times Z \times \mathcal{D} \mathcal{M}^{p}(\Omega \backslash D)$

The definition of the space $\mathcal{D} \mathcal{M}^{p}(\Omega \backslash D)$ is recalled in Remark 3.10 and the application of ALG2 algorithm for the augmented Lagrangian $L_{r}$ can be found in [10]. The interested reader can check [9, 11, 14, 15, 13, 16, 5, 2] for more details on saddle-point problems and ALG2 algorithm.

4.3. Some examples. We take $\Omega=[0,1] \times[0,1]$ and $g=0$ on $\partial \Omega$. The first three examples are performed with a Finsler distance $d_{\sigma}$ of Riemannian type

$$
d_{\sigma}(x, y)=\inf _{\substack{\zeta \in \operatorname{Lip}([0,1] ; \bar{\Omega}) \\ \zeta(0)=x, \zeta(1)=y}} \int_{0}^{1} k(\zeta(t))|\dot{\zeta}(t)| \mathrm{d} t .
$$

For the first test we take $k(x, y)=1$ and $\rho^{+}=2, \rho^{-}=\delta_{(0.5,0.5)}$. 


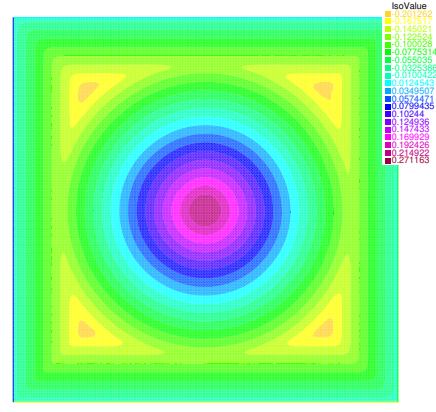

(A)

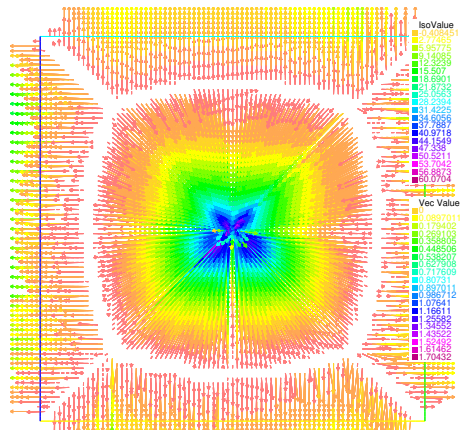

(B)

Figure 1. (A): the potential $u,(\mathrm{~B})$ : the flow $\phi$.

In the second test we take $\rho^{+}=4 \chi_{\left[(x-0.3)^{2}+(y-0.2)^{2}<0.03\right]}$ and $\rho^{-}=4 \chi_{\left[(x-0.7)^{2}+(y-0.8)^{2}<0.03\right]}$ and $k(x, y)=5-3 e^{-2 *\left((x-0.5)^{2}+(y-0.5)^{2}\right)}$.

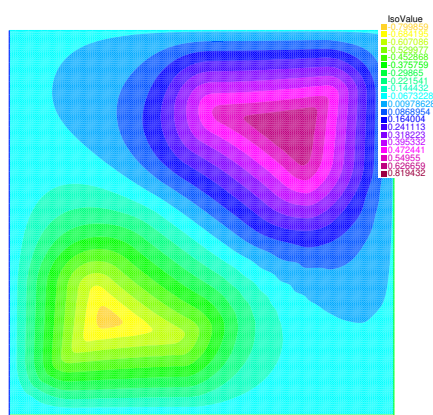

(A)

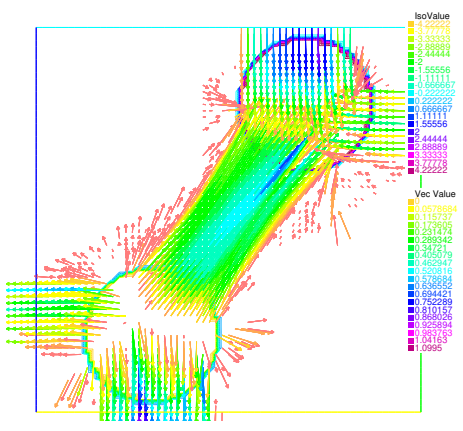

(в)

Figure 2. (A): the potential $u,(\mathrm{~B})$ : the flow $\phi$.

In the third test we take two Gaussian densities $\rho^{+}=e^{-40 *\left((x-0.75)^{2}+(y-0.3)^{2}\right)}$ and $\rho^{-}=$ $e^{-40 *\left((x-0.3)^{2}+(y-0.65)^{2}\right)}$. We change the distance $d_{\sigma}$ by taking a degenerate $k$. In particular, we choose

$$
k(x, y)=\sqrt{(1-2 x)^{2}\left(y-y^{2}\right)^{2}+(1-2 y)^{2}\left(x-x^{2}\right)^{2}} \chi_{\mathcal{B}}
$$

where $\mathcal{B}=\left\{(x, y) \in[0,1]^{2}: \sqrt{(x-0.5)^{2}+(y-0.5)^{2}}>0.25\right\}$. We clearly see that the flux is concentrated essentially on the region where $k$ vanishes, i.e, on $\mathcal{B}^{c}$, which in terms of optimal transport with respect to $d_{\sigma}$ represents a free transport region. 


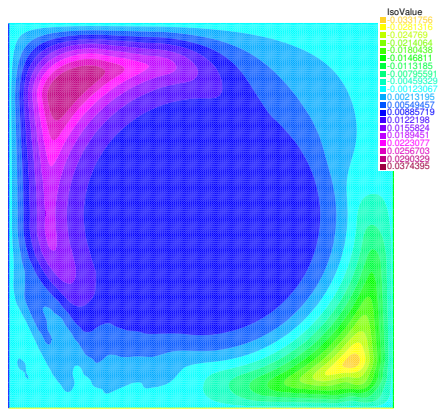

(A)

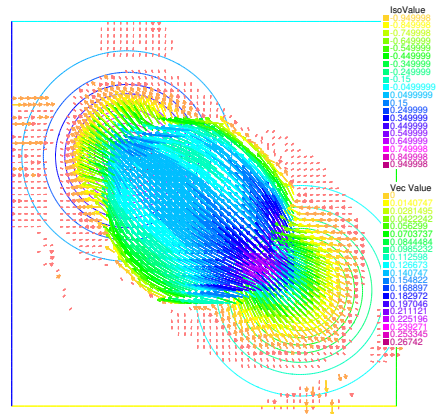

(B)

Figure 3. (A): the potential $u,(\mathrm{~B})$ : the flow $\phi$ privileging the zero set of $k$.

In the last test we consider a Finsler metric of cystalline type, namely

$$
\sigma(v)=\max _{i=1, \cdots, 5} v \cdot d_{i}
$$

with $d_{1}=(1,-1), d_{2}=(1,-0.8), d_{3}=(-0.8,1), d_{4}=(-1,1), d_{5}=(-1,-1)$.

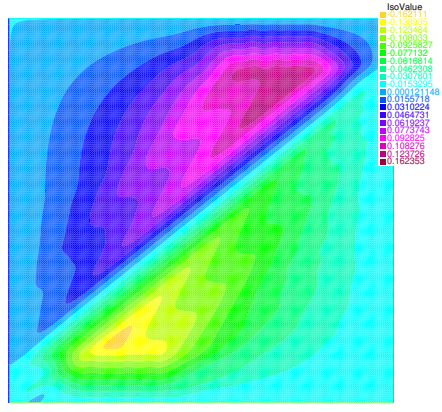

(A)

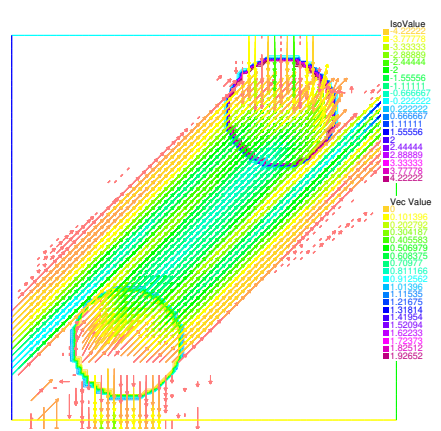

(B)

Figure 4. (A): the potential $u$, (B): the flow $\phi$.

\section{Comments And extentions}

A natural extension one can think of is the HJ equation with double obstacles on the boundary. More precisely one can consider the equation

$$
H(x, \nabla u)=0 \text { on } \Omega, g_{1} \leq u \leq g_{2} \text { on } \partial \Omega
$$

where $g_{i}: \partial \Omega \rightarrow \mathbb{R}$ are continuous functions satisfying the compatibility condition $g_{1}(x)-g_{2}(y) \leq$ $d_{\sigma}(y, x)$ for any $x, y \in \partial \Omega$.

In order to establish the link between this problem and a Bekmann-type problem, we consider as previously the following maximization problem

$$
\left(\mathcal{M}_{D}\right)_{o}: \max \left\{\int_{\Omega} u \mathrm{~d} \rho: u \in W^{1, \infty}(\Omega), \sigma^{*}(x, \nabla u) \leq 1 \text { and } g_{1} \leq u \leq g_{2} \text { on } \partial \Omega\right\} .
$$


Similarly, we can state the following result.

Theorem 5.12. The optimization problem $\left(\mathcal{M}_{D}\right)_{o}$ coincides with the following Beckmann-type problem, denoted by $(\mathcal{B K})_{o}$,

$\min _{\phi \in \mathcal{M}_{b}(\bar{\Omega})^{N}, \nu \in \mathcal{M}_{b}(\partial \Omega)}\left\{\int_{\Omega} \sigma\left(x, \frac{\phi}{|\phi|}(x)\right) \mathrm{d}|\phi|+\int_{\partial \Omega} g_{2} \mathrm{~d} \nu^{+}-\int_{\partial \Omega} g_{1} \mathrm{~d} \nu^{-}:-\operatorname{div}(\phi)=\rho-\nu\right.$ in $\left.\mathcal{D}^{\prime}\left(\mathbb{R}^{N}\right)\right\}$.

Moreover, $u$ and $(\phi, \nu)$ are optimal solutions to $\left(\mathcal{M}_{D}\right)_{o}$ and $(\mathcal{B K})_{o}$, respectively if and only if

$$
\begin{cases}-\operatorname{div}(\phi)=\rho-\nu & \text { in } \mathcal{D}^{\prime}\left(\mathbb{R}^{N}\right) \\ \phi(x) \cdot \nabla_{|\phi|} u(x)=\sigma\left(x, \frac{\phi}{|\phi|}(x)\right) & \text { for }|\phi|-\text { a.e. } x \\ g_{1} \leq u \leq g_{2} \text { on } \partial \Omega & \text { and } u=g_{1} \text { for } \nu^{-}-\text {a.e. } x \text { and } u=g_{2} \text { for } \nu^{+}-\text {a.e. } x .\end{cases}
$$

Sketch of proof. We define on $\mathcal{C}(\partial \Omega) \times \mathcal{C}(\partial \Omega)$ the following functional on $\mathcal{C}(\partial \Omega) \times \mathcal{C}(\partial \Omega)$ by, for $(v, w) \in \mathcal{C}(\partial \Omega) \times \mathcal{C}(\partial \Omega)$,

$$
E(v, w)=-\sup \left\{\int_{\Omega} u \mathrm{~d} \rho: u \in \operatorname{Lip}(\Omega), \sigma^{*}(x, \nabla u(x)) \leq 1, g_{1} \leq u+v, u+w \leq g_{2} \text { on } \partial \Omega\right\} .
$$

Most of the arguments of Section 3 can be reproduced to show that $E$ is convex and lower semicontinuous, which gives $E(0,0)=E^{* *}(0,0)$. It follows that $\max \left(\mathcal{M}_{D}\right)_{o}=\min (\mathcal{B K})_{o}$. We now turn to the optimality conditions. First observe that, for any feasible $u$ and $(\phi, \nu)$, we get

$$
\begin{aligned}
\int_{\bar{\Omega}} u \mathrm{~d} \rho & =\int_{\Omega} \nabla_{|\phi|} u(x) \frac{\phi}{|\phi|}(x) \mathrm{d}|\phi|+\int_{\bar{\Omega}} u \mathrm{~d} \nu \\
& \leq \int_{\Omega} \sigma\left(x, \frac{\phi}{|\phi|}(x)\right) \mathrm{d}|\phi|+\int_{D} g_{2} \mathrm{~d} \nu^{+}-\int_{\partial \Omega} g_{1} \mathrm{~d} \nu^{-},
\end{aligned}
$$

where we have used $\nabla_{|\phi|} u(x) \phi(x) \leq \sigma\left(x, \frac{\phi}{|\phi|}(x)\right)$ for $|\phi|$ - a.e. $x$ by the fact that $u$ is 1-Lipschitz w.r.t. $d_{\sigma}$.

On the other hand, $u$ and $(\phi, \nu)$ are optimal for $\left(\mathcal{M}_{D}\right)_{o}$ and $(\mathcal{B K})_{o}$ respectively, if and only if

$$
\int_{\Omega} u \mathrm{~d} \rho=\int_{\Omega} \sigma\left(x, \frac{\phi}{|\phi|}(x)\right) \mathrm{d}|\phi|+\int_{\partial \Omega} g_{2} \mathrm{~d} \nu^{+}-\int_{\partial \Omega} g_{1} \mathrm{~d} \nu^{-},
$$

i.e. the equality holds in (5.16), which is equivalent to the system of optimality conditions as desired.

Let us mention that problems of the form $\left(\mathcal{M}_{D}\right)_{o}$ and $(\mathcal{B K})_{o}$ can arise when studying optimal transport problems with some import/export costs on the boundary. In the case where the transport cost is given by the Euclidean distance $c(x, y)=|x-y|$ (in our case when considering the Eikonal equation $|\nabla u|=1$ ) we refer the reader to [20]. 


\section{REFERENCES}

[1] Martin Beckmann. A continuous model of transportation. Econometrica: Journal of the Econometric Society, 20(4): 643-660, 1952.

[2] Jean-David Benamou and Guillaume Carlier. Augmented Lagrangian methods for transport optimization, mean field games and degenerate elliptic equations. Journal of Optimization Theory and Applications, 167(1):1-26, 2015.

[3] Jean-David Benamou, Guillaume Carlier, and Roméo Hatchi. A numerical solution to Monge's problem with a Finsler distance as cost. ESAIM: Mathematical Modelling and Numerical Analysis, 52(6):2133-2148, 2018.

[4] Guy Bouchitté and Guiseppe Buttazzo. Characterization of optimal shapes and masses through MongeKantorovich equation. Journal of the European Mathematical Society, 3(2):139-168, 2001.

[5] Stephen Boyd, Neal Parikh, Eric Chu, Borja Peleato, and Jonathan Eckstein. Distributed optimization and statistical learning via the alternating direction method of multipliers. Foundations and Trends@ in Machine Learning, 3(1):1-122, 2011.

[6] Michael G. Crandall, Hitoshi Ishii, and Pierre-Louis Lions. User's guide to viscosity solutions of second order partial differential equations. Bulletin of the American mathematical society, 27(1):1-67, 1992.

[7] Andrea Davini. Smooth approximation of weak Finsler metrics. Differential and Integral Equations, 18(5): 509-530, 2005.

[8] Luigi De Pascale and Chloé Jimenez. Duality theory and optimal transport for sand piles growing in a silos. Advances in Differential Equations, 20(9-10):859-886, 2015.

[9] Jonathan Eckstein and Dimitri P Bertsekas. On the Douglas-Rachford splitting method and the proximal point algorithm for maximal monotone operators. Mathematical Programming, 55(1-3):293-318, 1992.

[10] Hamza Ennaji, Nouredine Igbida and Van Thanh Nguyen. Augmented Lagrangian method for HamiltonJacobi equtions. submitted, 2020.

[11] Ivar Ekeland and Roger Temam. Convex Analysis and Variational Problems. Classics in Applied Mathematics, SIAM, 1999.

[12] Albert Fathi and Antonio Siconolfi. PDE aspects of Aubry-Mather theory for quasiconvex Hamiltonians. Calculus of Variations and Partial Differential Equations, 22(2):185-228, 2005.

[13] Michel Fortin and Roland Glowinski. Augmented Lagrangian methods: applications to the numerical solution of boundary-value problems, volume 15. Elsevier, 2000.

[14] Roland Glowinski and J Tinsley Oden. Numerical methods for nonlinear variational problems. 1985.

[15] Ronald Glowinski and Patrick Le Tallec. Augmented Lagrangian and operator-splitting methods in nonlinear mechanics, volume 9. SIAM, 1989.

[16] Noureddine Igbida and Van Thanh Nguyen. Augmented Lagrangian method for optimal partial transportation. IMA Journal of Numerical Analysis, 38(1):156-183, 2018.

[17] Noureddine Igbida and Van Thanh Nguyen. Optimal partial mass transportation and obstacle Monge-Kantorovich equation. Journal of Differential Equations, 264(10): 6380-6417, 2018.

[18] Noureddine Igbida and José M Mazón, and Julio D Rossi, and Julián Toledo, Optimal mass transportation for costs given by Finsler distances via p-Laplacian approximations, Advances in Calculus of Variations, 11(1): 1-28, 2018.

[19] Pierre-Louis Lions. Generalized solutions of Hamilton-Jacobi equations, volume 69. Pitman, London, 1982.

[20] José M. Mazón and Julio D. Rossi and Julián Toledo, An optimal transportation problem with a cost given by the Euclidean distance plus import/export taxes on the boundary. Revista Matemática Iberoamericana, 30(1): 277-308, 2014.

[21] Filippo Santambrogio. Optimal transport for applied mathematicians. Calculus of variations, PDEs, and modeling, volume 87. Cham: Birkhäuser/Springer, 2015.

[22] Antonio Siconolfi. Hamilton-Jacobi equations and Weak KAM Theory, pages 4540-4561. Mathematics of Complexity and Dynamical Systems, 2011.

[23] Cédric Villani. Optimal transport: old and new, volume 338. Springer Science \& Business Media, 2009. 